\title{
Avaliação das madeiras maranhenses para uso em baquetas de baterias
}

\author{
David Guilhon ${ }^{1}$; \\ Kamylla Baldez Figueiredo2; \\ Alan Carlos Serra Silva3;
}

resumo:

Como artífice da narrativa da história, estão os instrumentos musicais e seus acessórios que tal qual os demais artefatos humanos evoluíram às custas de tentativa e erro na criação e fabricação, sendo também um marco divisor entre povos primitivos e os civilizados. As baquetas surgiram como extensões das habilidades do instrumentista. Atualmente, o conhecimento científico aliado aos modernos processos de produção delineiam as qualidades e fabricação de instrumentos musicais e seus acessórios, no caso deste estudo, a baqueta. Dentro dessa seara, a madeira é o material atualmente mais usado para a fabricação destes acessórios, em especial a Nogueira Americana, em virtude de suas propriedades. Assim, este artigo visa a investigação de madeiras locais para a fabricação de baquetas.

Como metodologia de pesquisa, realizou-se a análise comparativa entre as madeiras tradicionalmente usadas para a fabricação de baquetas com as espécies maranhenses aptas ao comércio. As propriedades tecnológicas adotadas foram: a densidade, módulo de elasticidade, módulo de ruptura à flexão, dureza Janka e coeficiente de anisotropia. Investigou-se também a trabalhabilidade. Estabeleceram-se níveis de enquadramento para os gráficos gerados por cada propriedades em níveis de proximidade com as espécies mais usadas (Nogueira Americana, Carvalho Branco, Pau-Marfim e Bordo-açucareiro).

Os resultados investigados mostram uma tendência pela preferência de espécies mais densas, elásticas, duras e resistentes a impactos, bem como com grã direita, facilidade de pintura e torneamento; sobretudo, ao observar-se as madeiras mais usadas. Dessa forma, apresentam-se as melhores madeiras em ordem decrescente: Pau-d'arco, Cumaru, Maçaranduba, Jatobá, Sucupira, Maracatiara, Piquiá e Goiabão. Deve-se lembrar que não é o resultado definitivo, mas as sugestões enquadradas pela literatura sobre a possibilidade de uso e aplicação das madeiras maranhenses para baquetas. Portanto, ainda são necessárias mais pesquisas para o aprofundamento no assunto, como ensaios destrutivos com as melhores espécies e produção de protótipos para testes em situação de uso.

palavras-chave:

Design; instrumentos musicais; madeiras maranhenses; baquetas.

Espaço reservado para organização do congresso.

\footnotetext{
${ }^{1}$ http://lattes.cnpq.br/8935299354649314

${ }^{2}$ http://lattes.cnpq.br/3187968009897948

${ }^{3}$ http://lattes.cnpq.br/7797181208816681
} 


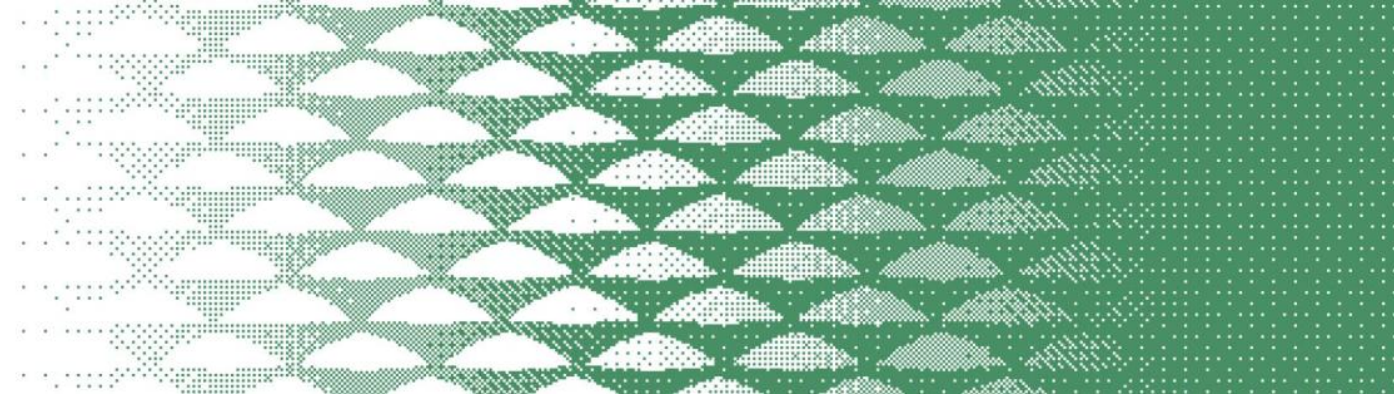

\section{Introdução}

A música acompanha o homem ao longo de sua trajetória, de modo que, para Rosauro (2016), a natureza e sua percepção do mundo foram suas principais referências rítmicas, sobretudo os sons emitidos por insetos, pássaros e outros animais motivaram tais simulações em outros aparatos. O mesmo autor sugere o caótico cenário pré-histórico como o berço dos instrumentos percussivos, onde o desenvolvimento de práticas religiosas que orbitavam em torno destas condições ecoa em sons de batidas dos pés no chão, das mãos contra o corpo e outros rudimentares artefatos (pedaços de madeira, ossos, pedras e chocalhos).

Assim, a descoberta da música é o divisor de águas entre povos primitivos e civilizações antigas, partindo de ruídos a produção consciente de sons mais refinados (ANDRADE, 2015). Esse caminho longo, onde a arte das musas ajuda a contar tal história, passa pelo processo evolutivo dos instrumentos musicais, que para Morris (2010), assim como o desenvolvimento de artefatos, é uma atividade natural e própria do homem, ocorrendo de forma intuitiva, valendo-se dos erros de processo e produção como critérios eliminatórios. A importância que os instrumentos musicais possuem é notória, pois, Bucur (2016) percebe-os como objetos culturais, já que seu timbre apresenta características de um período geohistórico. Entretanto, é recente a consciência de um entendimento projetual e científico como princípios construtivos destes artefatos.

Binder (2006) ressalta que a forma de tocar dos primeiros bateristas foi inicialmente influenciada pela forma militar, que se valia de técnicas como os rolls, flares, ruffs e outros rudimentos. Mesmo não batendo nos pratos, já usavam suas baquetas na caixa. Hashimoto (2003) também lembra o uso festivo destes acessórios com pontas de madeiras para execução ao ar livre. Logo, é por meio do par de baquetas que realiza o som na bateria. Braga (2011) adiciona a responsabilidade dela, um bastão de madeira com uma das pontas arredondadas, como mediadora dos movimentos da mão à superfície das peles dos tambores e dos pratos. Sobretudo, quando se trata no atual cenário, onde a bateria tem espaço cativo em diversos gêneros musicais, sendo um instrumento "globalizado".

Por tratar-se de um acessório importante, onde basicamente, só se consegue extrair o som por meio delas, as baquetas precisam ser bastante resistentes aos impactos e ter durabilidade, já que inúmeras vezes suas pontas são batidas na parte central ou periférica das peles dos tambores da bateria (caixa, tons e surdo), bem como nos aros destes com sua parte mais mediana e em pratos de bronze. Então, o material que traduz tais necessidades é a madeira, sobretudo a Nogueira (Carya spp) que é a espécie preferida pelos grandes fabricantes internacionais (BARROS, 2020; MULTISOM, 2020). A combinação de densidade e elasticidade refletem na sonoridade que os músicos desejam.

Entendendo que a flora maranhense é dotada de uma gama de espécies e aptas para fins comerciais, bem como já se esboçam usos de outras espécies brasileiras como o Roxinho, como madeiras alternativas, pretende-se com este trabalho fazer uma avaliação comparativa das espécies maranhenses com as madeiras mais usadas no mundo para a fabricação de baquetas.

\section{Baquetas: anatomia e tipos de pontas}

A profusa existência de marcas e modelos, com uma gama de combinações das partes de sua anatomia (BRAGA, 2011), como mostra o Gráfico 1. A escolha da baqueta é pautada no conforto, estilo musical e sonoridade desejada. A baqueta geralmente tem, em média, $39 \mathrm{~mm}$ de diâmetro e $130 \mathrm{~mm}$ de comprimento. Para Braga (2011), o peso e dimensões da baqueta, bem como o tipo de madeira e sua densidade, tem influência no timbre do som. O referido autor observa a mesma ocorrência com o tipo de ponta, por ser o ponto de contato da baqueta com a peça da bateria (pele ou pratos). Dessa forma, ressalta que baquetas leves e com pontas com menor ponto de contato "realçam os harmônicos superiores, tornando o timbre mais penetrante e agressivo" (BRAGA, 2011, p.12). Barsalini (2014) 


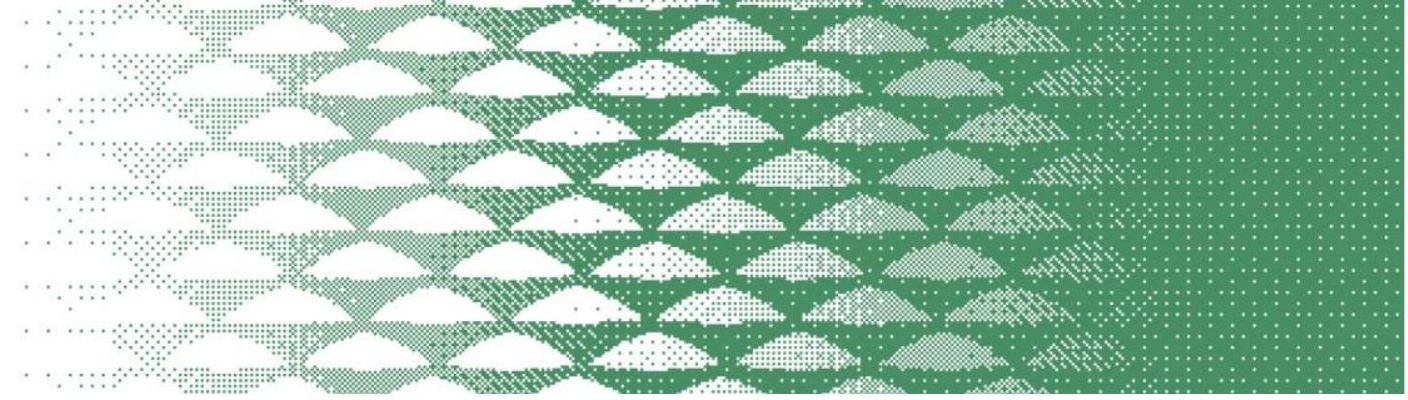

complementa a esse discurso o controle dinâmico e expressivo que cada baterista possui - o swing - e as características de cada bateria, também como fatores para a escolha da baqueta.

\section{Anatomia da baqueta}
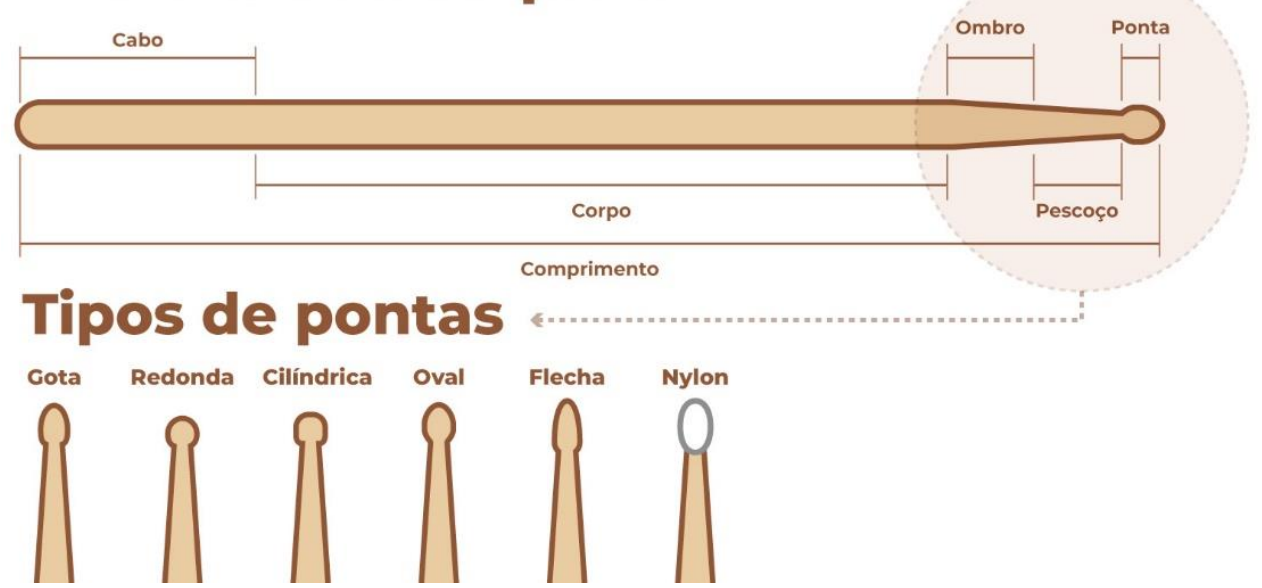

Gráfico 1 - Anatomia da baqueta e tipos de pontas.

O cabo, com medida-padrão de $130 \mathrm{~mm}$, proporciona o conforto ao musicista em sua performance. Já o corpo da baqueta é a parte que a define em termos dimensionais o acessório, que também indica o gênero musical a qual será destinada, além da resistência mecânica e elasticidade (LIVERPOOL, 2020). Em seguida, vê-se o afunilamento e ligação à ponta é parte do ombro e pescoço, que, para Braga (2016), há uma relação indireta entre a sua espessura e a agilidade de execução que a baqueta promove durante a peça musical. Por fim, desemboca-se na ponta, a ponto de ter-se vários tipos que são destinados a diferentes estilos musicais, técnicas e relacionado ao nível de perícia do baterista. Barros (2020) apresenta, por exemplo, a ponta redonda como opção de som uniforme, indicadas para iniciantes. Enquanto indica, ainda, a ponta barril para gravações em estúdio e apresentações em lugares abertos, pois favorece um ganho de volumes nos pratos por conta da maior área de contato. E sugere a ponta gota para músicos mais experientes, pois esta confere mais versatilidade.

Além disso, há categorização das baquetas que converge várias destas características, principalmente o diâmetro dela. $\mathrm{O}$ indicativo de algarismos e letras aponta relação indireta com a espessura do acessório (quanto maior o número, menor o diâmetro). Dessa forma, Barros (2020) apresenta quatro tipos principais: 2B (a mais espessa), 5A (para iniciantes), 5B e 7A (a menos espessa). A relação do diâmetro com a madeira usada (atentando-se especialmente à densidade) acontece de forma indireta. Em outras palavras, madeiras menos densas se destinam para séries de maior diâmetro, como $2 \mathrm{~B}$ e as mais densas são empregadas em séries com menor diâmetro, como a 5B e 7A. Essa correspondência externa a versatilidade e a sonoridade desejadas.

\section{Metodologia de trabalho}

Este artigo pauta-se no estudo comparativo entre espécies tradicionalmente usadas em baquetas de baterias (padrão) e madeiras maranhenses. A lista das madeiras-padrão, conforme os oito maiores fabricantes mundiais do artefato (BARROS, 2020) que são: D'Addario, Liverpool, Meinl, Pro Orca, Stagg, Tama, Vater e Vic Firth; e no estudo de Souza (2020) e Meier (2020). Da mesma forma, segue a lista das madeiras maranhenses com as mesmas propriedades, seguindo os estudos de Vasconcellos et al. (2001) e Nahuz et al. (2013). 


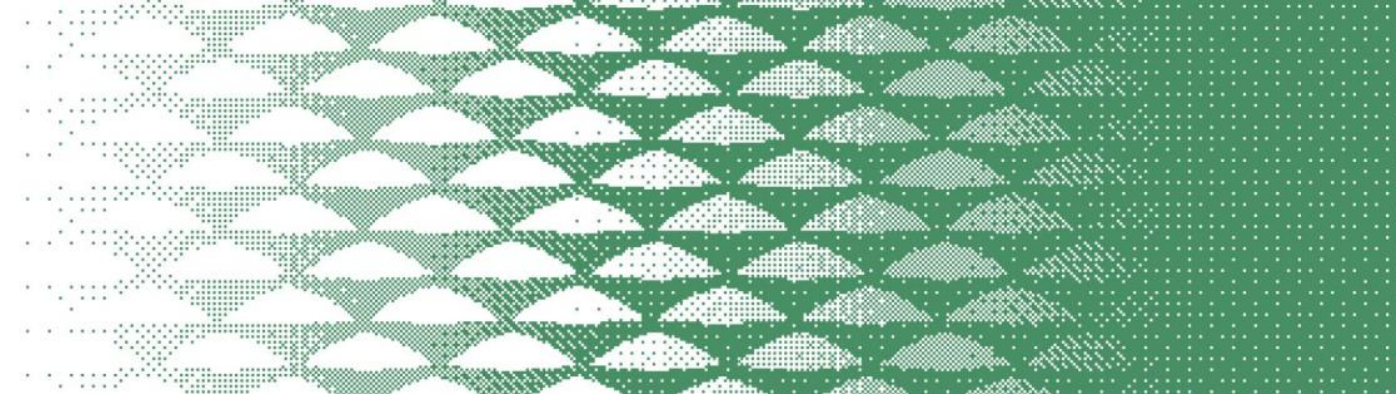

A literatura descreve as seguintes propriedades tecnológicas usadas para instrumentos musicais que norteiam este trabalho que: a densidade ( $\rho$ ), módulo de elasticidade (E), módulo de ruptura à flexão (G), dureza Janka (Hj) e o coeficiente de anisotropia (WEGST, 2006; KÚDELA; KUNŠTÁR, 2011; BENNETT, 2016, SPROßMANN et al., 2017).

A partir dos valores destas propriedades, geraram-se 5 gráficos, onde o primeiro é de dispersão acerca do cruzamento entre densidade $(\rho)$ e módulo de elasticidade (E), já que é um fator indicativo das qualidades acústicas do material. Neste gráfico trabalha-se, ainda, com elipse de confiança 95\%, resultante dos índices das madeiras-referência, criando um espectro para fins de análise. Desse modo, estabelece-se um critério classificatório que lista 4 níveis, que são: semelhantes às padrão, próximas das padrão, dentro do espectro e fora do espectro. Já os outros quatro gráficos são de colunas. Estes mostram cada uma das demais propriedades e trabalha-se com duas instâncias classificatórias: acima da faixa e dentro da faixa. Para todos os gráficos, a cor azul representa as madeiras-referência e a vermelha retrata as espécies locais. Por fim, vem a trabalhabilidade como fator classificatório e eliminatório, visando observar a facilidade ou não do processamento da madeira durante a fabricação, desembocando na viabilidade técnica.

\section{Resultados e discussões}

Avaliou 23 espécies de madeiras maranhenses, sendo comparadas com 12 tipos de madeiras tradicionalmente usadas para a fabricação de baquetas para bateria.

Assim, as espécies tradicionalmente usadas são: Bétula amarela (BT), Bétula branca (BB), Bétula doce (BD), Bordo Açucareiro (BO), Cárpino (CP), Carvalho Branco (CB), Granadillo (GR), Nogueira (NO), Pau-marfim (PM), Pau-rosa (PR), Toona (TO) e Wenge (WE). As características tecnológicas destas madeiras estão catalogadas pelo The Wood (2020).

Já as espécies analisadas são: Angelim (AG), Bacuri (BA), Cachaceiro (CA), Cedro (CD), Cedrorana (CE), Cerejeira-da-Amazônia (CJ), Copaíba (CP), Cumaru (CM), Curupixá (CX), Freijó (FR), Goiabão (GO), Guanandi (GU), Jatobá (JA), Louro-cravo (LC), Louro-rosa (LR), Maçaranduba (MÇ), Maracatiara (MR), Mogno (MG), Pau-d'arco (PD), Piqui (PI), Piquiá (PQ), Sucupira (SU) e Tatajuba (TA).

Sobre as propriedades avaliadas, observa-se a relação entre o módulo de elasticidade (E) e a densidade ( $\rho$ ) da madeira, pois, para Bennett (2016), aponta características acústicas como a velocidade do som O módulo de elasticidade aprecia o nível de capacidade que o material tende a voltar ao seu estado natural sem deformar após a aplicação de uma força externa (OLIVEIRA; DELLA LUCIA; VITAL, 1988). Já a densidade relaciona-se diretamente com a resistência que o mesmo material tem quando tal força externa é sobre ele aplicado, figurando que materiais mais densos tendem a ser mais resistentes. Sendo assim, Soares (2014) menciona a ligação entre as características vibracionais e de absorção do som com a densidade, apontando que madeiras menos densas caminham para maior absorção sonora, enquanto as mais densas apresentam configurações vibracionais mais elevadas.

O Gráfico 2 apresenta justamente o que Wegst (2006) em seu estudo confrontou: módulo de elasticidade $(E)$ e a densidade $(\rho)$ da madeira. $O$ referido autor percebeu que tais dados apontam para a aplicação em instrumentos musicais. Assim, buscou-se verificar o nível de semelhança destes valores entre as madeiras. O Pau d'arco e a Nogueira, conhecida pelo seu nome inglês de Hickory no mercado, apresentam valores muito próximos, evidenciando notória similaridade. De igual modo, observa-se a relação entre o Curupixá e a Toona e, sensivelmente menos elástico e mais denso, Mogno. Também se percebe similaridade entre o Cachaceiro e a Bétula Amarela. Condições semelhantes também são encontradas entre o Bacuri e o Bordo Açucareiro e o Goiabão e o Bordo Açucareiro, Cárpino e Carvalho 


\section{JOP 21 DESIGN

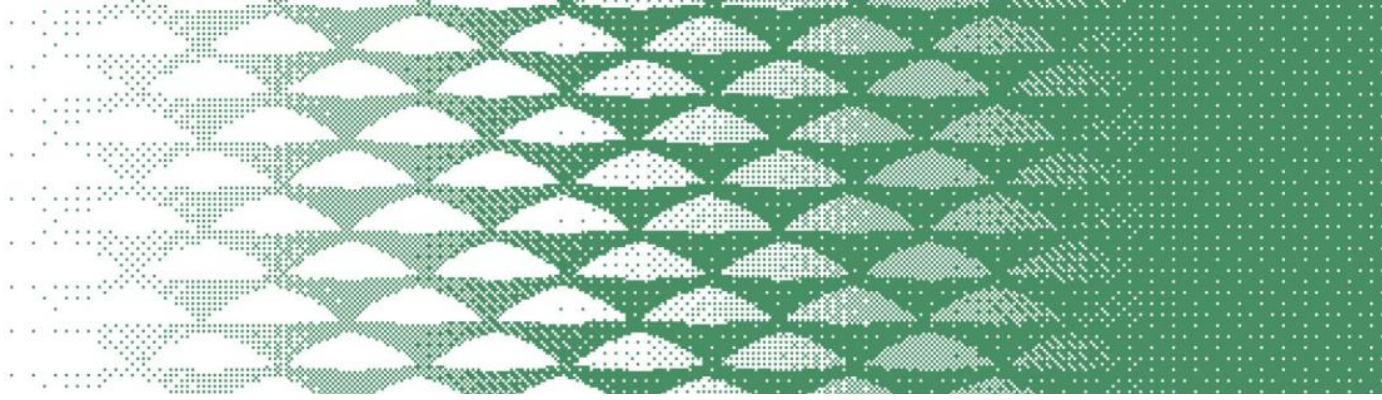

atingem os pratos e chimbal, peças estas feitas de bronze. Estas técnicas do meio baterístico citadas por Barsalini (2014) buscam a liberdade do baterista e a fidelidade de reprodução do som de outros instrumentos percussivos se enquadram, sobretudo, na verificação da dureza Janka, que é um dado que mede a resistência à penetração de objetos sólidos em sua estrutura direções normal e paralela às fibras (XAVIER, 2008). Silva Filho, Rocha e Moura (1992) atestam a estreita e direta relação desta grandeza com a densidade.

A respeito do módulo de ruptura à flexão, percebe-se que no Gráfico 3 as madeiras nativas têm valores abaixo das referências. Contudo, se levar-se em consideração apenas as madeiras mais usadas pelos fabricantes (Nogueira, Pau-Marfim, Bordo-Açucareiro e Carvalho Branco), os resultados favorecem o enquadramento das espécies locais em duas instâncias: a) acima da faixa - Pau-d'arco e Sucupira e; b) dentro da faixa - Jatobá, Cumaru, Maçaranduba, Piquiá e Piqui. A média dos números destas madeiras avaliadas é 3,90\% maior, se comparadas com a seleta lista dos fabricantes. Vê-se que ao examinar-se também o menor índice da madeira-referência - a Toona - amplia-se a quantidade de madeiras regionais a ponto de não serem enquadradas apenas o Angelim, Cerejeira, Guanandi, Cedrorana e Curupixá (todas com valores abaixo de 71,5 MPa).

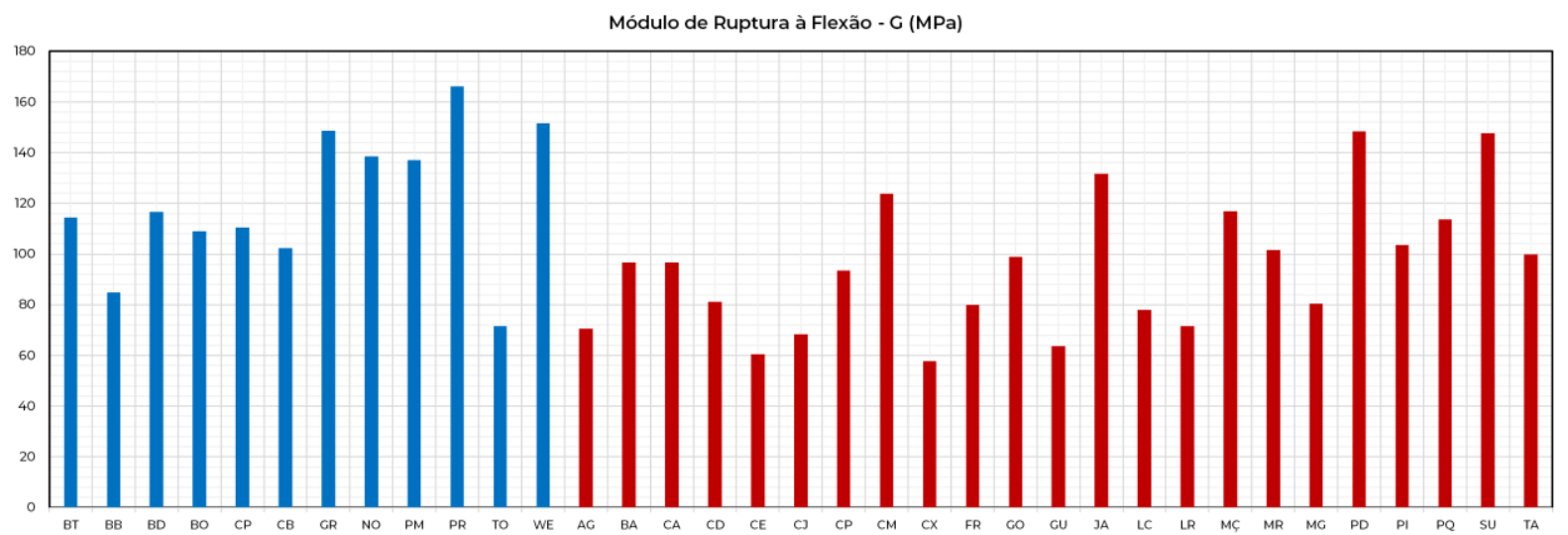

Gráfico 3 - Comparação do módulo de ruptura à flexão entre as espécies tradicionalmente usadas e as maranhenses.

O impacto em si que a baqueta sofre ao bater contra a estrutura das peças da bateria, bem como seu uso contínuo, leva ao desgaste com arranhões, marcas, fissuras, rachaduras, desfragmentação e até a quebra do acessório. Sendo assim, a dureza Janka observa o quanto as madeiras dessas baquetas precisam resistir a esse tipo de degradação externa de teor invasivo. Para tanto, o Gráfico 4 traz a comparação entre espécies, confirmando que as tidas como padrão possuem, em média, 15,16\% mais resistentes que as nativas. Mesmo considerando apenas as madeiras mais utilizadas pelos fabricantes, ainda assim são 12,50\% mais robustas. Mas, adotando estas espécies como base de valores, obtêm-se, então, dois níveis: a) acima da faixa - Jatobá, Pau-d'arco, Cumaru e Maçaranduba; b) dentro da faixa - Sucupira, Goiabão, Maracatiara, Tatajuba, Bacuri e Cachaceiro. Já nessas condições, tais madeiras regionais superam, em média, $38,46 \%$ as mais usadas pela indústria. Se, ainda, delimitar-se ao menor valor da lista das espécies-referência, apenas o Louro-rosa, por uma diferença mínima de 1,73\%, não se enquadra e o Guanandi não apresenta dados específicos para tal propriedade. 


\begin{tabular}{|c|c|c|c|c|c|c|c|c|c|c|c|c|c|c|c|c|c|c|c|c|c|}
\hline & \multicolumn{20}{|c|}{ RANQUEAMENTO } \\
\hline & & 1 & 2 & 3 & 4 & 5 & 6 & 7 & 8 & 9 & 10 & 11 & 12 & 13 & 14 & 15 & 16 & 17 & 18 & 19 & 20 \\
\hline \multirow{5}{*}{ 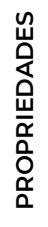 } & $E \times p$ & $\mathrm{CM}$ & PD & MÇ & JA & SU & CA & $\mathrm{PQ}$ & $\mathrm{PI}$ & MR & AG & $\mathrm{CX}$ & MG & TA & & & & & & & \\
\hline & G & PD & SU & JA & $\mathrm{CM}$ & $\mathrm{MÇ}$ & $\mathrm{PQ}$ & $\mathrm{PI}$ & MR & TA & GO & BA & CA & $\mathrm{CP}$ & CD & MG & FR & LC & LR & & \\
\hline & $\mathrm{Hj}$ & JA & PD & $\mathrm{CM}$ & $\mathrm{MÇ}$ & su & GO & TA & BA & CA & $\mathrm{PQ}$ & AG & MG & PI & LC & CE & $\mathrm{CP}$ & FR & CX & CJ & CD \\
\hline & Anisot & PD & SU & CM & $\mathrm{MÇ}$ & $\mathrm{PQ}$ & BA & CA & MR & $\mathrm{PI}$ & JA & & & & & & & & & & \\
\hline & Trab & GO & MÇ & JA & PD & $\mathrm{CM}$ & MR & $P Q$ & & & & & & & & & & & & & \\
\hline
\end{tabular}

Gráfico 6-Quadro-resumo dos melhores resultados para as propriedades avaliadas.

Como crivo final do estudo, apresenta-se aqui os melhores resultados em ordem decrescente: Pau-d'arco, Cumaru, Maçaranduba, Jatobá, Sucupira, Maracatiara, Piquiá e Goiabão. Estes podem ser apontados como baquetas dos tipos 5B e 7A. As que se encontram dentro do espectro da pesquisa podem ser trabalhadas como baquetas para iniciantes e estudo, em séries $2 \mathrm{~B}$ e $5 \mathrm{~A}$. As demais não se enquadram no perfil esperado para baquetas, podem apresentar sérios problemas ou durante o uso por parte do baterista ou até durante a fabricação, tornando-se opções inviáveis como acessório de instrumentos musicais.

É interessante lembrar que este artigo não apresenta um estudo definitivo, mas sugere, dentro da literatura, a possibilidade de uso e aplicação das madeiras maranhenses para baquetas. Para tanto, sugerem-se que sejam feitos ensaios destrutivos com os melhores resultados, bem como protótipos para serem avaliados em situação de uso por bateristas profissionais e definições por tipos de baquetas.

\section{Evaluation of Maranhão woods for the manufacture of drum sticks}

Abstract: Musical instruments and their accessories are the creator of the narrative of history, so I ate the other human artifacts that evolved at the expense of trial and error in creation and manufacture, being also a dividing point between primitive and civilized peoples. The drumsticks emerged as extensions of the instrumentalist's skills. Currently, scientific knowledge combined with modern production processes define the qualities and manufacture of musical instruments and their accessories, in the case of this study, the drumstick. Within this area, wood is currently the most used material for the manufacture of these accessories, especially American Hickory, due to its properties. Thus, this article aims to investigate local woods for the manufacture of drumsticks.

A comparative analysis was carried out between the woods traditionally used for the manufacture of drumsticks with the species from Maranhão suitable for trade as a research methodology. The technological properties adopted were: density, Young's modulus, flexural rupture modulus, Janka hardness and anisotropy coefficient. Workability was also investigated. Framing levels were established for the graphics generated by each property at levels of proximity to the most used species (American Hickory, White Oak, Ivory and Hard Maple).

The results point to a tendency towards the preference of denser, elastic, harder and impact resistant species, as well as with the right grain, easy painting and turning; above all, when the most used wood is used as a parameter. Soon, the best woods are presented in descending order: Ipe, Cumaru, Massaranduba, Courbaril, Sucupira, Tigerwood, Pekea and Abiurana. It is interesting to note that it is not the definitive result, but the suggestions framed by the literature on the possibility of using and 
applying Maranhão woods for drumsticks. Therefore, more research is still needed to deepen the subject, such as destructive tests with the best species and production of prototypes for tests in use situations.

Keywords: Design; musical instruments; Maranhão woods; drumsticks

\section{Referências bibliográficas}

ANDRADE, M. Pequena história da música. Rio de Janeiro: Nova Fronteira, 2015.

BARROS, A. Top 10 Melhores Baquetas para Comprar em 2021. Mybest - Serviço de Recomendações | Acessórios para Instrumentos Musicais. 2020. Disponível em: https://mybestbrazil.com.br/11653. Acessado em: 30 mar 2021.

BARSALINI, L. Modos de execução da bateria no samba. Tese (Doutorado em Música) Programa de Pós-Graduação em Música do Instituto de Artes. Universidade Estadual de Campinas. 264 p. Campinas, 2014.

BENNETT, B. C.; The Sound of Trees: Wood Selection in Guitars and Other Chordophones, in: Economic Botany, $X X(X)$. New York: 2016. p. 1-15.

BINDER, F. P. Bandas Militares no Brasil: difusão e organização entre 1808-1889 - volume I. Dissertação (Mestrado em Música). Programa de Pós-Graduação em Música do Instituto de Artes. Universidade Estadual Paulista. São Paulo. 135 p. 2006.

BRAGA, Tarcísio. A caixa clara na bateria: Estudo de caso de performances dos bateristas Zé Eduardo Nazário e Marcio Bahia. Dissertação (mestrado em Música) - Universidade Federal de Minas Gerais, Escola de Música. Belo Horizonte. 119 p. 2011.

BUCUR, V. Handbook of Materials for String Musical Instruments. Springer International Publishing: Switzerland, 2016.

COSTA, J. A. Classificação de madeiras da Amazônia para composição de instrumento musical de corda através da técnica de excitação por impulso. Dissertação (Mestrado em Ciências Florestais e Ambientais). Universidade Federal do Amazonas, Manaus, 2017.

HASHIMOTO, Fernando Augusto de Almeida. Análise musical de "estudo para instrumentos de percussão", 1953, M. Camargo Guarnieri; Primeira peça escrita somente para instrumentos de percussão no Brasil. Dissertação (mestrado. Universidade Estadual de Campinas, Instituto de Artes. 143 p. Campinas, 2003.

KÚDELA, J.; KUNŠTÁR, M. Physical-acoustical characteristics of maple wood with wavy structure. In: Annals of Warsaw University of Life Sciences - SGGW. Forestry and Wood Technology. No 75, 2011: p 12-188.

MEIER, E. The Wood Database: Explore Woods. 2020. Disponível em: https://www.wooddatabase.com/ Acessado em: 15 mar 2020.

MORESCHI, J. C. Propriedades tecnológicas da madeira. Departamento de Engenharia e Tecnologia Florestal, Universidade Federal do Paraná, Curitiba, 2009.

MORRIS, R. Fundamentos de Design de Produto. Porto Alegre: Bookman, 2010.

MULTISOM. Qual tipo de baqueta escolher? Saiba o que levar em consideração. 2017.

Disponível em: https://blog.multisom.com.br/tipos-de-baquetas/ Acessado em: 30.mar.2020. 
NAHUZ, M. A. R.; MIRANDA, M. J. A. C.; IELO, P. K. Y.; PIGOZZO, R. J. B.; YOJO, T. Catálogo de madeiras brasileiras para a construção civil. São Paulo: IPT - Instituto de Pesquisas Tecnológicas do Estado de São Paulo, 2013.

OLIVEIRA, J. T. S.; DELLA LUCIA, R. M.; VITAL, B. R. Estudo das propriedades físicas e tecnológicas da madeira da pindaíba (Xylopia sericea St. Hil.). In: Revista Árvore, v.12, n.2, p. 129-133, 1988.

SILVA FILHO, Danilo Fernandes da; ROCHA, Jadir de Souza; MOURA, José Brandão de. Influência da densidade na dureza Janka em oito espécies madeireiras da Amazônia Central. In: Acta Amazônica, 22 (2): 275 - 283.1992.

SPROBMANN, R.; ZAUER, M.; WAGENFÜHR, A. Characterization of acoustic and mechanical properties of common tropical woods used in classical guitars, in: Results in Physics, v. 7. p. 1737-1742. 2017.

ROSAURO, N. História dos instrumentos sinfônicos de percussão - Da antiguidade aos tempos modernos. Santa Maria: UFSM, 2016.

VASCONCELLOS, F.J.; FREITAS, J.A.; LIMA, V.M.O.C.; MONTEIRO, L.V.; PEREIRA, S.J. Madeiras Tropicais de Uso Industrial no Maranhão: características tecnológicas. Instituto Nacional de Pesquisas da Amazônia (INPA), Universidade Federal do Maranhão (UFMA), Manaus, 2001. 96 p.

WEGST, U. G. K. Wood for sound. In: American Journal of Botany 93(10). 1439-1448. 2006.

XAVIER, Rafael Borba Lopes. Avaliação da dureza Janka, densidade e estabilidade de quatro espécies de Eucalyptus implantadas no Estado do Rio de Janeiro. Monografia (Bacharel em Engenharia Floresta) - Instituto de Florestas da Universidade Federal Rural do Rio de Janeiro.

Seropédica, 31 p. 2008. 